\title{
ENHANCING WIRELESS SPECTRUM UTILIZATION WITH A CELLULAR-AD HOC OVERLAY ARCHITECTURE
}

\author{
Srivatsan Sankaranarayanan, Panagiotis Papadimitratos, and Amitabh Mishra* \\ Bradley Department of Electrical and Computer Engineering \\ Virginia Polytechnic Institute and State University, Blacksburg, USA \\ \{srivatsan, papadp, mishra\}@vt.edu
}

\begin{abstract}
The spectrum of deployed wireless cellular communication systems is found to be under-utilized, even though licensed spectrum is at a premium. In this paper, we design a system with an ad hoc overlay network, which we denote as the secondary system (SEC), to efficiently utilize the bandwidth left unused in a cellular system, which we denote as the primary system (PRI). The basic design principle is that the SEC operates in a non-intrusive manner and does not interact with the PRI. We develop the AS-MAC, an Ad hoc SEC Medium Access Control protocol to enable the interoperation of the PRI-SEC system. We address a number of technical challenges pertinent to this networking environment, and investigate a number of $A S-M A C$ variants. Our performance evaluation results indicate that AS-MAC can transparently utilize up to $80 \%$ bandwidth left unused by the PRI.
\end{abstract}

\section{INTRODUCTION}

There is a strong belief that the spectrum both in the public as well as private sector in the United States is getting scarce. Recent measurements for cellular systems in major metropolitan areas ([1], [2]) suggest that spectrum utilization in several frequency bands is very low for extended periods of time. This means that the primary cause of spectrum scarcity is its inefficient utilization, rather than the unavailability of resources. It also suggests that adoption of efficient modulation and coding techniques, which can clearly improve spectrum utilization, cannot alone address the inefficiency.

A promising approach, known as spectrum sharing or pooling [3], is to enable two systems accessing the same spectrum. The owner of the spectrum, which we denote as the primary system $(P R I)$, can allow a secondary system $(S E C)$ to operate in the same spectrum, under the assumption that $S E C$ utilizes only the portion of the spectrum left unused by the PRI. One example of such a scenario is that of a cellular provider leasing its unused spectrum to a $S E C$ when the cellular traffic is expected to be significantly lower, e.g., between 9PM and 7AM. The SEC could, for

\footnotetext{
* This work was supported by DARPA/Raytheon Company for the XG program under contract No. 12292
}

example, utilize the unused resources to offer wireless Internet access services for home users.

In this paper, we consider the design of a $S E C$ system overlaid on a $P R I$ cellular system. In particular, we assume that that the PRI is a TDMA/FDMA based GSM cellular network [14]. The SEC is a multi-hop ad hoc network, which we denote as the Ad hoc Secondary Network ( $A S N)$. The fundamental constraints that $A S N$ has to respect are $(i)$ the $A S N$ operate only over the resources (i.e., bandwidth) left unutilized by the PRI GSM, (ii) the operation of the $A S N$ leads to no performance degradation of the $P R I$, and (iii) there is no exchange of signaling information between the $P R I$ and the $A S N$.

To enable such an approach, we propose here the $A d$ hoc SEC Medium Access Control (AS-MAC) protocol, which is responsible for the following basic tasks. First, it detects the frequency bands utilized by the entities of the $P R I$, i.e., base station $(B S)$ and the mobile stations ( $M S$ 's). Then, $A S-M A C$ detects and maintains a picture of the (portion of) $P R I$ resources that remain unutilized. Finally, with this information at hand, $A S-M A C$ provides a flexible facility for the $A S N$ nodes ( $A N \mathrm{~s}$ ) to use those resources for their communication, while satisfying the above-mentioned constraints $(i)-(i i i)$.

The contribution of this paper is the identification of technical challenges in the development of a PRI-SEC system, and a practical solution proposed based on the $A S$ $M A C$ protocol. Our evaluation of the protocol indicates that $A S-M A C$ enables the $A S N$ to efficiently utilize up to $80 \%$ of the otherwise unused bandwidth of the GSM PRI in a single-hop scenario. Moreover, when the $A S N$ operates across a multihop topology, bandwidth reuse multiplies the benefit of the $A S N$ deployment our performance evaluation section shows.

In the rest of the paper, we first provide an architectural view of the proposed system, identify the technical challenges therein, and discuss the basic ideas of our approach to address those challenges. The $A S-M A C$ protocol is defined next, followed by its performance evaluation. Finally, we discuss related schemes in the literature and conclude with a discussion of future work. 


\section{SYSTEM ARCHITECTURE AND OVERVIEW}

An example of the physical architecture of the PRI$S E C$ system is illustrated in Figure 1: within the GSM system, $M S^{\prime}$ s communicate with the $B S$, while $A N \mathrm{~s}$ form a multi-hop, peer-to-peer topology within the same GSM cell. Within a $G S M$ cell, a set, $C$, of channel pairs, that is, frequency bands is allocated for use by the $B S$ and $M S^{\prime}$ s, out of $C_{\text {total }}=124$ available $G S M$ bands [14]. For each pair, one channel is used for $B S$ to $M S$ (downlink) and one channel for $M S$ to $B S$ (uplink) communication. Each up- or down-link is divided into $T_{S}$ time slots. The $B S$ transmits on dedicated slots in the downlink channel, the Frequency Correction Channel $(F C C H)$ and Synchronization Channel $(S C H)$, signals to enable the $M S$ 's to achieve time synchronization with the $B S$. These are signaling channels and are point-to-multipoint.

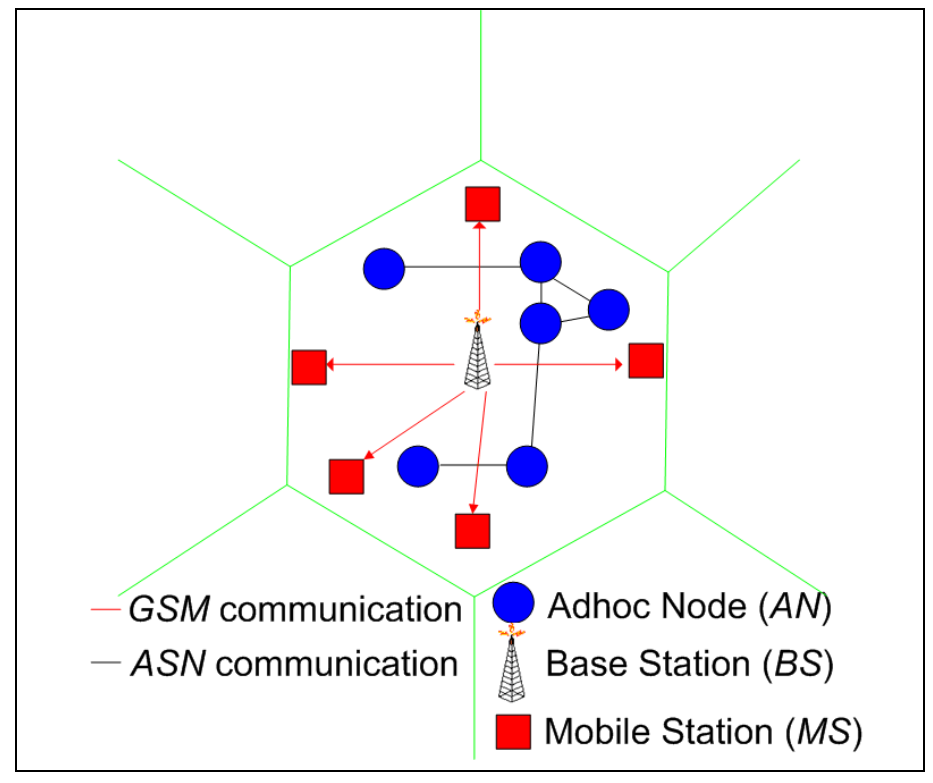

Figure 1. System diagram

Each of the $A N s$ within an $A S N$, needs to first detect the communication structure of the $P R I$, and then identify the available resources, which are the time-slots within each of the cell's frequency bands. Then, the $A N \mathrm{~s}$ utilize this available bandwidth to communicate, without interfering with the operation of the PRI. We note that the $A S N$ can operate across multiple cells, yet we leave this as future work.

The first challenge for $A S N$ is to detect the PRI communication structure and identify the available resources. To do so, we assume that $A N s$ are equipped with a sensing module, that is, hardware that provides the capability for wide-band spectrum sensing [15], [19], [20]. For our system, it suffices that the sensing module detects the presence of a signal (that is, energy level above a threshold) within each of the $C$ bands. The $A N$ s equipped with the sensing module first detect the $C$ bands in use in the cell.
Then, they obtain the slots boundaries (i.e. the beginning and end of each time-slot) by decoding the $\mathrm{FCCH}$ and $\mathrm{SCH}$ signaling. Finally, the sensing module is used to construct an up-to-date map of available time slots. With a complete picture of the slot availability on the downlinks, $A N \mathrm{~s}$ can communicate among themselves. More importantly, by transmitting during slots sensed and guaranteed to be idle, $A N \mathrm{~s}$ ensure that there will be no collision with or obstruction of the PRI traffic.

Note that only the resources on the downlinks are utilized by the system described in this paper, as determining the boundaries of the slots in the uplinks would require the collaboration of the PRI (i.e., the $B S$ ). We assume that $A N$ s use the GSM physical layer, below the FDMA/TDMA $G S M$ medium access to communicate among themselves within the $A S N$.

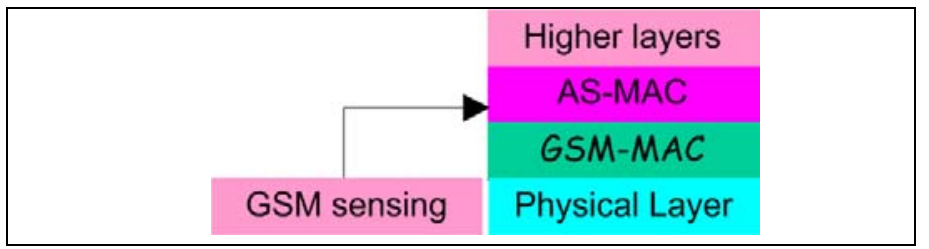

Figure 2. Protocol stack of $A N$

The solution we are after seeks to enable any network protocol stack in the $A S N$. Nonetheless, the challenge lies in transmitting a packet from the $A S N$ network across the available spectrum, and dependent only on the $P R I$ operation and traffic resources. To achieve this goal, a protocol that acts as an intermediary between the $A S N$ network layer and the primary GSM system is necessary. Essentially, such a protocol acts as a medium access control protocol from the point of view of the $A S N$. Yet, it is not truly a medium access control $(M A C)$ protocol, as it operates on top of the GSM MAC protocol. We denote this protocol as Ad hoc Secondary Medium Access Control (AS-MAC). Figure 2 illustrates the $A S N$ protocol stack.

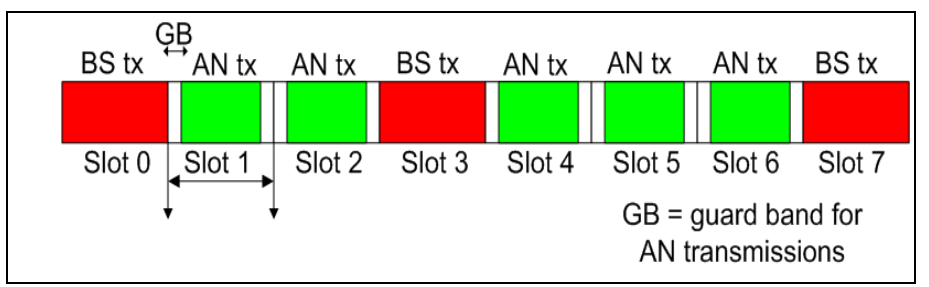

Figure 3. GSM Slot Utilization by ASN nodes

The $A N \mathrm{~s}$ have only one transceiver, while multiple $G S M$ channels are available. As a result, the $A S-M A C$ provides for the selection of one among those channels. To do so, a handshake is necessary between the sender and the receiver: the sender provides candidate channels and the receiver selects a desirable channel. Then, not only the two 
nodes but also their neighbors can be aware of the channel in use. This exchange of information is performed across a commonly agreed channel, which we denote as the control channel $(C C)$, while the actual data transmission takes place across the remaining data channels.

Finally, once the data channel is selected, $A S-M A C$ has to actually transmit the data. The challenge here is that the transmission has to take place within the available slots. Essentially, to ensure non-interference with the PRI, ANs 'hijack' free GSM slots, once it is definite that the slot is not utilized. Figure 3 shows a single time-slotted GSM downlink consisting of eight slots numbered 0 to 7 , with slots 0,3 , and 7 used by the PRI. Since the occupancy (availability) of slots depends on the $P R I$ traffic, time progress of the $A S N$ protocol, in our case $A S-M A C$, must take place only when $P R I$ slots are free. Otherwise, the state of the $A S N$ protocol must essentially freeze. For example, in Figure 3 , if a message transmission is to occupy three slots, starting from Slot 1, then, counting those slots must 'stop' during Slot 3. In general, the $A S N$ packets are larger than the number of bits that can be transmitted in a single GSM slot. Thus, the sender needs to fragment data and send it over successive free slots which may not be consecutive.

\section{AS-MAC PROTOCOL OPERATION}

First, we discuss how ANs make use of their sensing hardware, to identify and use $P R I$ channel and unused slots. Then, we present the operation of our $A S-M A C$ protocol, and finally discuss additional implementation aspects of the protocol.

\section{A. SENSING AND CHANNEL USAGE}

$A N s$ identify the GSM downlinks in a PRI cell basically through sensing and the following steps. First, the $A N \mathrm{~s}$ scan the PRI bands to determine the $\mathrm{FCCH}$ and $\mathrm{SCH}$ signaling channels, detecting the specific transmission pattern of those channels [14]. This way, ANs obtain the timing information of the slot boundaries. With this information in hand, $A N \mathrm{~s}$ scan again the set of $P R I$ channels specified to be used as downlinks, searching for those in use within the cell. To determine if indeed a downlink is in use, $A N \mathrm{~s}$ sense within the boundaries only. If the sensed signals fit the slots, $A N$ infers that this signal is transmitted by $B S$.

Note that it is possible that a node receives signals from multiple base stations, for example, when it is close to the boundary of two cells. By measuring the received signal strength, similarly to the RSSI measurements [14] performed by the mobile nodes, the node can classify the signals. Yet, it is possible that the in-use channel information is not the same across all $A N s$. This can be somewhat detrimental to the performance of the $A S N$, but as it will become clearer below, the network can operate as pairs of sender-receiver $A N$ s communicate always on a mutually agreed data channel.

Among the available channels, $A N \mathrm{~s}$ follow a convention to choose the downlink GSM control channel, exclusively for $A S-M A C$ control traffic. In our design, the downlink that bears the $\mathrm{FCCH}$ and $\mathrm{SCH}$ signaling is the one utilized as the $A S-M A C$ control channel. This is the first downlink channel identified by $A N$ s and any new $A N$ joining the $A S N$ within the cell can unambiguously identify it. The set of remaining channels, denoted as $C_{d}$, are used for data traffic.

At all times, $A N s$ determine whether a given slot is free. To ensure that a slot is indeed left unused by the PRI, AN's sense the all (downlink) channels during a period of time $\tau$ at the beginning of each slot. It suffices that $\tau$ is of the order of $5 \mu \mathrm{s}$, after the $G S M$ guard band $(15 \mu \mathrm{s})$. Overall, the required sensing time (after the guard band) is a small fraction of $T$ the GSM slot duration of $577 \mu$ s. Through the sensing operation, $A N s$ build and dynamically update a data structure, $p$ Usage which maintains statistics of the PRI slot usage history, with more recent sample having higher weights. This information is used in dynamically selecting the preferred data channels for packet transmission. Nonetheless, such preference does not guarantee that the slot availability will remain as estimated, or does imply that any prediction of future usage is made. Instead, the sensing module is utilized at all slot boundaries to actually determine the slot availability.

It is straightforward to utilize the sensing module, which is utilized only for $\tau$ to sense PRI traffic, for sensing of $A S N$ transmission. It suffices to activate the sensing module for a $\tau_{S E C}$ after the primary signal sensing. We denote this a secondary sensing, performed both on the $A S N$ 's control and data channels. Due to secondary and the control traffic, as explained below, $A N$ 's maintain sUsage, a data structure indicating the data channels is currently in use by other $A N$ 's.

\section{B. $A S$-MAC DESCRIPTION}

With the resource availability information at hand, $A S$ $M A C$ enables communication between any two neighboring $A N \mathrm{~s}$. Basically, $A S-M A C$ provides the means for nodes to first agree upon a data channel, through a handshake that involves the exchange of three control messages, a Request To Send (RTS), a Clear To Send (CTS), and a Reservation (RES) message transmitted in this order. Our experiments, presented in the performance evaluation section showed that the RES message may not be necessary. As a result, we identify and discuss two versions of $A S-M A C$, one which uses $R E S$ and we denote as $A S-M A C_{l}$, and one without $R E S$ denoted as $A S-M A C_{2}$. Since the latter is found more efficient, we discuss this variant below, referring to 
$A S-M A C_{1}$ and $A S-M A C_{2}$ interchangeably unless otherwise noted.

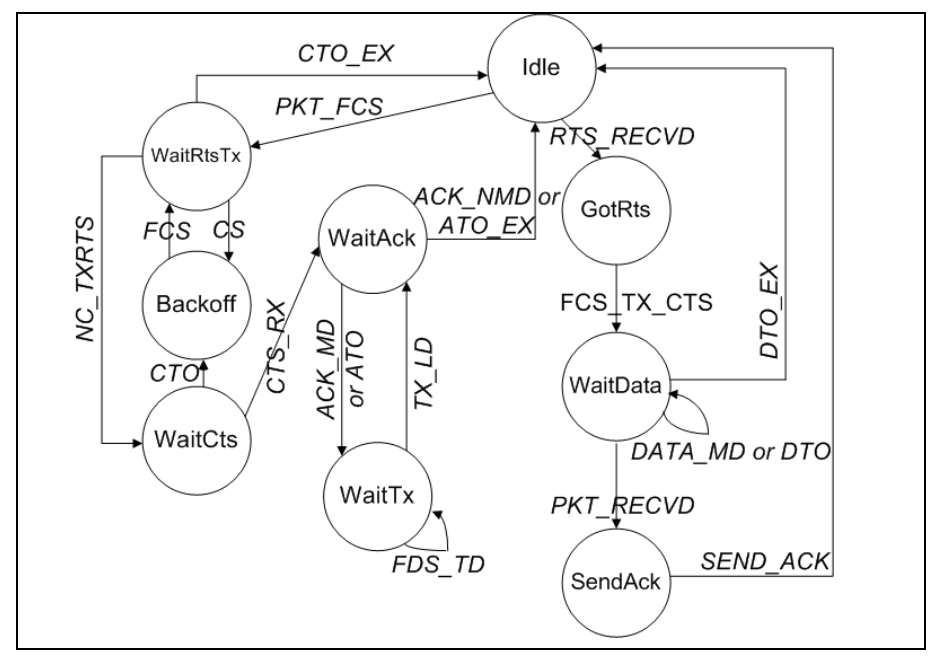

Figure 4: $A S-M A C$ state transition diagram

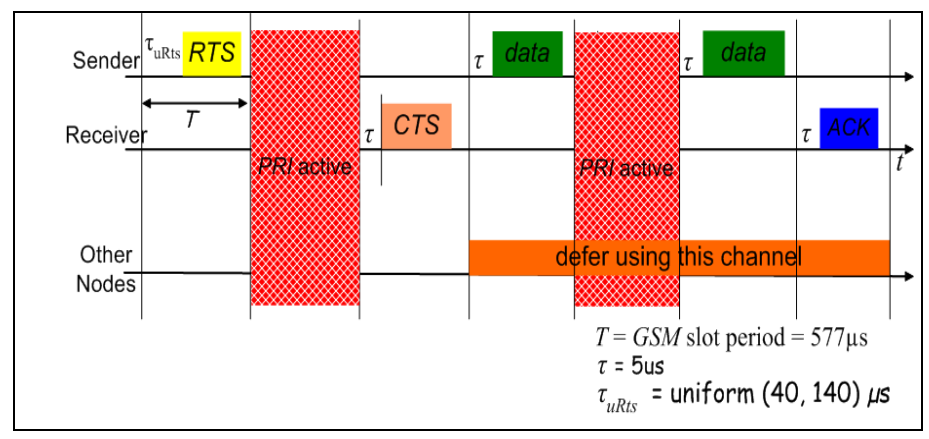

Figure 5. $A S-M A C$ packet transfer

The finite state diagram in Figure 4 defines the $A S$ $M A C$, with Table 1 explaining the conditions and actions for each transition. Figure 5 illustrates the $A S-M A C$ operation.

When an $A N$ has an eligible packet to transmit, it waits for a free control slot. A packet is eligible for transmission if the destination is not currently involved in communication with some other node as indicated by CTS and RES received by the $A N$. It then schedules a unicast $R T S$ transmission after $\tau_{u R t s}$ which is uniform in a window $W_{\text {uRts. }}$ This is done to introduce some randomness in RTS transmissions so that collisions among $R T S$ are reduced. $W_{\mathrm{uRts}}$ is set to $(40 \mu \mathrm{s}$ to 140$) \mu \mathrm{s}$ from slot beginning. When the scheduled waiting time for $R T S$ transmission expires, $A N$ senses the control channel. If it is found busy, $A N$ retries the $R T S$ in the next free control slot without incrementing the backoff counter.

If no carrier is sensed, $A N$ sends $R T S$ as shown in Figure 5. The RTS contains a bit map of channel status from the perspective of the sender, the number of slots needed to transmit the packet (called $N A V$ ). After sending
$R T S$, the sender waits until the end of the next free slot on the selected data channel to receive CTS. This is an important point because, if the sender were to wait for just one slot duration and if the next slot were to be used by GSM, the receiver will not transmit CTS. Then the sender would timeout unnecessarily. Similar phenomenon happens for all timeouts in the protocol.

\begin{tabular}{|c|c|}
\hline Condition/Action Id & Description \\
\hline$P K T_{-} F C S$ & $\begin{array}{l}\text { Packet available and free control slot / } \\
\text { schedule } R T S \text { transmission }\end{array}$ \\
\hline CTO & Timeout / ++numCtsTimeouts \\
\hline$C T S \_R X$ & CTS received \\
\hline$C S$ & Carrier sensed \\
\hline$N C_{-} T X R T S$ & No carrier / Tx RTS \\
\hline$F D S \_T D$ & $\begin{array}{l}\text { Free data slot and more than one } \\
D A T A / \text { tx DATA }\end{array}$ \\
\hline$A C K \_M D$ & $A C K$ recvd and more $D A T A$ \\
\hline ATO & ACK timeout $/++$ numAckTimeouts \\
\hline$T X \_L D$ & $\begin{array}{l}\text { Free data slot and only one pending } \\
\mathrm{D} A T A / \mathrm{tx} D A T A\end{array}$ \\
\hline$A C K \_N M D$ & $A C K$ got and no more pending $D A T A$ \\
\hline$F C S$ & Free control slot \\
\hline SEND_ACK & Free data slot / send $A C K$ \\
\hline$A T O_{-} E X$ & $\begin{array}{l}\text { MAX_ACK_TIMEOUTS exceeded / } \\
\text { drop packet }\end{array}$ \\
\hline$F C S \_T X \_C T S$ & Free control slot / send CTS \\
\hline RTS_RECVD & Unicast $R T S$ recvd \\
\hline$P K T_{-} R E C V D$ & $\begin{array}{l}\text { Packet received completely / pass } \\
\text { packet to higher layer }\end{array}$ \\
\hline CTO_EX & $\begin{array}{l}\text { MAX_CTS_TIMEOUTS exceeded / } \\
\text { drop packet }\end{array}$ \\
\hline$D T O \_E X$ & $\begin{array}{l}\text { MAX_DATA_TIMEOUTS exceeded / } \\
\text { drop packet }\end{array}$ \\
\hline$D A T A_{-} M D$ & $\begin{array}{l}D A T A \text { fragment got, more } D A T A \\
\text { pending }\end{array}$ \\
\hline DTO & DATA timeout / numDataTimeouts ++ \\
\hline
\end{tabular}

\section{Table 1: $A S-M A C$ protocol conditions and actions}

If CTS is not received by the sender, backoff counter is incremented and RTS is retried in the next free control slot. Once MAX_RTS_ATTEMPTS are exceeded, the packet is dropped. On receipt of RTS, receiver sends CTS as shown in Figure 5 which contains the receiver's and sender's Id, $N A V$, and also the channel selected for communication. The receiver selects that data channel which is free both at the sender and at the receiver, and which has the maximum number of free slots available. 
On receipt of $C T S$, sender sends $R E S$ in the case of $A S$ $M A C_{l}$. This is depicted in Figure 5. RES contains sender and receiver Ids, NAV info, and the channel chosen for data transfer. Other nodes that receive (RES and) CTS, know that they should prohibit themselves from using the specified channel until at least $N A V$ number of free slots have passed by on the chosen data channel. RES and CTS also tell other ANs not to attempt to send an RTS to the sender or the receiver as they will be busy in a data transfer and therefore cannot receive RTS.

After the sender and receiver complete the RTS/CTS (RES) handshake, the sender fragments the packet and transmits the fragments successively on all the free slots on the data channel. Figure 5 and Figure 6 indicate this operation and also illustrate that $A S-M A C$ does nothing in a slot that is being used by $P R I$. Fragments are identified by a sequence number beginning from zero. An $A C K$ is expected by the sender when there are no more pending fragments to send. This is indicated to the receiver by setting the $A C K$ flag in the header. A FINAL flag is also set whenever the sender sends the last fragment of the packet. $A C K$ from the receiver contains a bitmap acknowledging the fragment $I d s$ received in the current cycle (cycle refers to the time period in which one train of fragments is sent by the sender and an $A C K$ is sent by the receiver).

Here it is interesting to note that the sender does not reserve a channel for any fixed duration of time as is the case with 802.11 and Multi-channel MAC $(M M A C)$ protocols in general. This will not work because, the secondary cannot know the future channel/slot usage of the primary, so it has no way of telling when it will be done transmitting. Thus $A S-M A C$ uses a count of the number of free slots that is required for transmitting the data packet as the $N A V$. Third party $A N S$ that receive the CTS and RES, decrement the $N A V$ counter only when a free slot passes by on the selected data channel.

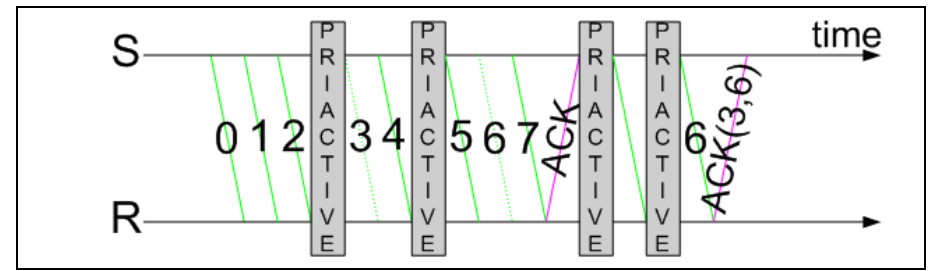

Figure 6. $A S-M A C$ error recovery process

On receipt of $A C K$, the sender updates its knowledge of successfully received fragments and retransmits only the unsuccessful fragments. When the sender sends the last pending fragment it always expects an $A C K$. This process is continued until the entire packet is transferred. An example error recovery situation is illustrated in Figure 6 for the case of a packet consisting of eight fragments numbered 0 to 8 . Fragments 3 and 6 are lost (shown in dotted lines). The first $A C K$ acknowledges all fragments except 3 and 6 which are then retransmitted in the next cycle and the packet transfer is completed.

On receipt of a packet with the FINAL flag set, the receiver knows that the last fragment has been received. Thereafter, on receipt of every fragment, the receiver checks to see if it has then received all the fragments. If so, the entire packet has been received and is passed on to the higher layer.

One problem arises when a large packet needs to be transmitted. $A C K$ packet needs to fit into one slot, so there is an upper bound on the number of bits available for acknowledging received fragments which means that the receiver cannot acknowledge an arbitrarily large number of fragments. In this case, the sender restricts the number of fragments to be sent in a cycle to a suitable value. The remaining fragments and any fragment not received successfully in the current cycle are transmitted in the next cycle.

\section{AS-MAC IMPLEMENTATION CONSIDERATIONS}

Next, we discuss issues related to the ASN transceivers. First, consider the transceiver turnaround time, that is, the period of time needed for a transceiver to switch from transmitting to receiving mode and vice-versa. In our system, such transitions need to occur at the $P R I$ slot boundaries. The aggregate time of the GSM guard band $(15 \mu \mathrm{s})$, the PRI sensing period $(5 \mu \mathrm{s})$, and the margin of $27 \mu \mathrm{s}$ to ensure negligible interference on $P R I$ transmissions, is well above the $802.11 \mathrm{~g}$ receive-to-transmit and transmitto-receive turnaround times of $5 \mu \mathrm{s}$ and $10 \mu \mathrm{s}$ for its $D H S S$.

Another concern is the time needed to dynamically switch a transceiver to different channels at different points in time. In $A S \_M A C$ such switching needs to take place after a RTS-CTS handshake and after the transmission of a packet when the sender and receiver want to switch to the control channel. The channel switching time allowed in 802.11 is $224 \mu \mathrm{s}$. Thus it seems impractical in the near future to achieve switching times less than about $45 \mu s$. To overcome this problem we suggest that both the sender and receiver freeze their operation in the next slot (irrespective of whether it is free or not) after the RTS-CTS handshake and resume the protocol operation thereafter. This allows ample time (at least full slot duration of $577 \mu \mathrm{s})$ to switch the transceiver to the chosen data channel.

It is important that time synchronization of $A S N$ with $B S$ be maintained all throughout. This necessitates $A N S$ to update their time reference by listening to the $F C C H$ and $S C H$ messages from $B S$ periodically. ANs could do this a few times a second whenever they are not transmitting a packet. It may be noted that MSs get such timing information from BS twice per second (when a call is in progress). 


\section{PERFORMANCE EVALUATION}

We evaluate the performance of our system, studying the improvement in spectrum utilization due to the $A S N$. We denote the $\%$ of bandwidth utilized by the $P R I$ when deployed alone as $P R I_{U}$, and \% of bandwidth utilized by the $P R I$ and the $A S N$ when both are deployed as $P R I A S N_{U}$. We quantify this improvement with two metrics: (i) the spectrum utilization improvement, $S U I=\left(P R I \_A S N_{U}-P R I_{\underline{U}}\right) / P R I_{U}$, and (ii) the utilization of available bandwidth $B U$, calculated as the fraction of the bandwidth used by $A N s$ over the PRI left-unused downlink bandwidth. Both $S U I$ and $B U$ are calculated as averages over the total duration of the simulation. $B U$ quantifies the effectiveness of our $A S-M A C$ and $S U I$ provides the overall picture of efficient utilization. Our results indicate that $A S$ $M A C$ is effective, with $B U$ up to $83 \%$, thus yielding up to $S U I$ of $40 \%$ in a single-hop setting and even more in multihop setting due to spatial reuse.

We use Qualnet [17] for the simulations of a single-hop as well as a 10 -by-10 grid topology of $100 \mathrm{ANs}$, with transmission and carrier sensing range set to $250 \mathrm{~m}$ and $625 \mathrm{~m}$ respectively. The capture threshold and the required SINR for successful reception are set to $10 \mathrm{~dB}$; ambient noise is assumed to be negligible, but errors are caused by interference. All ANs are within one cell of the PRI GSM system, with $C=8$ channel pairs, in use within the cell. One of the channels (the one with the lowest index) is assumed to be the control channel for $A N \mathrm{~s}$, and the remaining 7 channels are denoted as data channels. The PRI traffic occupies one or more time slots within each channel. We assume that the slot occupancy (availability) changes slowly compared to a packet transmission, as call holding times are in the order of few tens of seconds to minutes [14]. We vary the $\%$ of available slots in the control and data channels, with values from $25 \%$ ( 2 out of 8 slots per GSM frame) to $100 \%$ ( 8 out of 8 slots), denoting the $\%$ of available slots in each control and data channel as $B_{C}$ and $B_{D}$. The $A N s$ operate in saturation conditions, always having a packet to send. $A N S$ randomly select a neighbor to transmit a packet, with size fixed at 280 bytes including the $U D P$ and $I P$ headers. We show the performance of the two versions of $A S-M A C$ we discussed above, $A S-M A C_{I}$ and $A S-M A C_{2}$.

The main objective of $A S-M A C$ is to improve spectrum utilization. Recall however that, in the system evaluated here, the $A S N$ utilizes only the downlinks. Thus, at most only half of the total amount of GSM bandwidth left unused can be utilized (assuming symmetric GSM traffic as in the case of voice calls). Table 2 shows the performance of $A S-M A C_{2}$ in a single-hop environment with 40 colocated nodes, as a function of $B_{C}$ while $B_{D}=50 \%$. $A S N$ improve the bandwidth utilization up to $41.6 \%$ when con- trol bandwidth availability is $B_{C}=100 \%$, amounting to $B U=83.2 \%$ and $S U I$ up to $41.6 \%$. As $B_{C}$ decreases, the control channel gradually becomes a bottleneck, yet $B U$ degrades gracefully to $70 \%$ for $B_{C}=25 \%$ and $S U I$ remains equal to $35 \%$.

\begin{tabular}{|l|c|c|c|c|c|c|c|}
\hline$\% B_{C}$ & 25 & 37.5 & 50 & 62.5 & 75 & 87.5 & 100 \\
\hline$\% B U$ & 70.0 & 80.2 & 82.8 & 82.6 & 83.2 & 82.8 & 83.2 \\
\hline$\% S U I$ & 35.0 & 40.1 & 41.4 & 41.3 & 41.6 & 41.4 & 41.6 \\
\hline
\end{tabular}

Table 2: Spectrum utilized by $A S N(\mathrm{AS} \mathrm{MAC})$ in a single hop network, as a function of $\% \overline{A C B}$ (available control bandwidth)

In a multi-hop $A S N, A S-M A C$ can perform even better due to spatial reuse of the available bandwidth. Thus in this case $S U I$ and $B U$ can be more than $\left(100 \%-P R I_{\mathrm{u}}\right)$. We now consider the multi-hop grid topology. Figure 7 shows $B U$ when $B_{D}=25 \%$ and $50 \%$, as a function of $B_{c}$. We do not take the control channel bandwidth into account in these calculations. In that case, the spectrum utilized by $A S N$ would be slightly less than what our graphs indicate, yet the trends will remain the same.

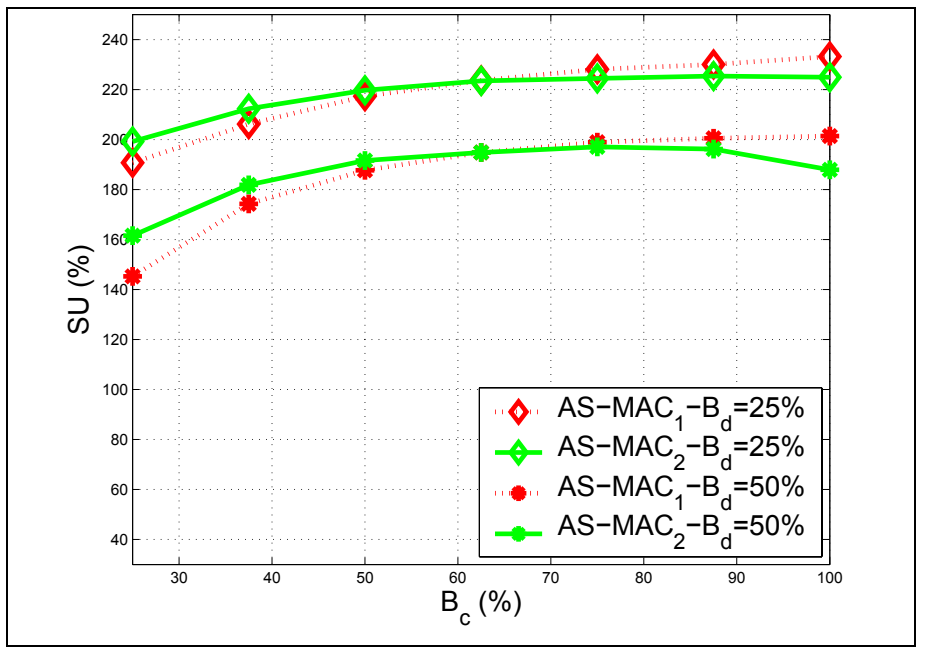

Figure 7. \% $B U$ when $B_{d}=25 \%$ and $50 \%$, Vs $B_{c}$

We observe that for lower values of $B_{c}$ (i.e. when control bandwidth is the bottleneck) $A S \_M A C_{2}$ performs the best as it needs less control bandwidth since it does not use $R E S$. But as $B_{c}$ is increased beyond about $60 \%, A S_{-} M A C_{l}$ starts performing better than $A S M A C_{2}$ as the control bandwidth is no more a bottleneck and the additional $R E S$ that $A S M A C_{1}$ uses brings in some benefits. But even when the available control bandwidth is $100 \%, A S \_M A C_{I}$ performs only marginally better than $A S S_{-} M A C_{2}$. In Figure 7, the comparison between $A S M A C_{1}$ and $A S M A C_{2}$ goes as $233.2 \%$ to $225 \%$ when $B_{d}=25 \%$ and $20 \overline{1} \%$ to $188 \%$ when $B_{d}=50 \%$. This means that the use of the additional $R E S$ control packet is not very useful. Note that $A S-M A C_{2}$ achieves $B U$ of about $188 \%$ when $B_{c}=100 \%$. Comparing this with the utilization in the single-hop case of $83 \%$ (as 
illustrated in Table 2 and the related discussion), we see that the gain due to spatial reuse in this case is about 2.5. Thus our protocol can perform significantly better in the multi-hop case than in the single-hop case.

When only one transceiver is available, the protocols suffer from the multi-channel hidden terminal problem $(M H T P)$ [6]. This means that the nodes will not be able to receive a significant number of control packets. This makes one think that the lack of utilization improvement when using RES is due to the nodes not being able to receive it rather than the additional RES not being effective. Thus we show the results when two transceivers are used by $A N s$ in Figure 8 when $B_{d}=25 \%$ and $B_{d}=50 \%$. Now there is no hidden terminal problem as one of the transceivers always listens to the control channel. Still we see that the performance achieved by $A S \_M A C_{1}$ compared to $A S \_M A C_{2}$ is still marginal (235\% to $230 \%$ when $B_{d}=25 \%$, and $211 \%$ to $203 \%$ when $B_{d}=50 \%$ ). Thus, it is evident that the use of an additional RES packet in the control handshake is not very useful when multi-channel sensing is available and therefore can be safely avoided.

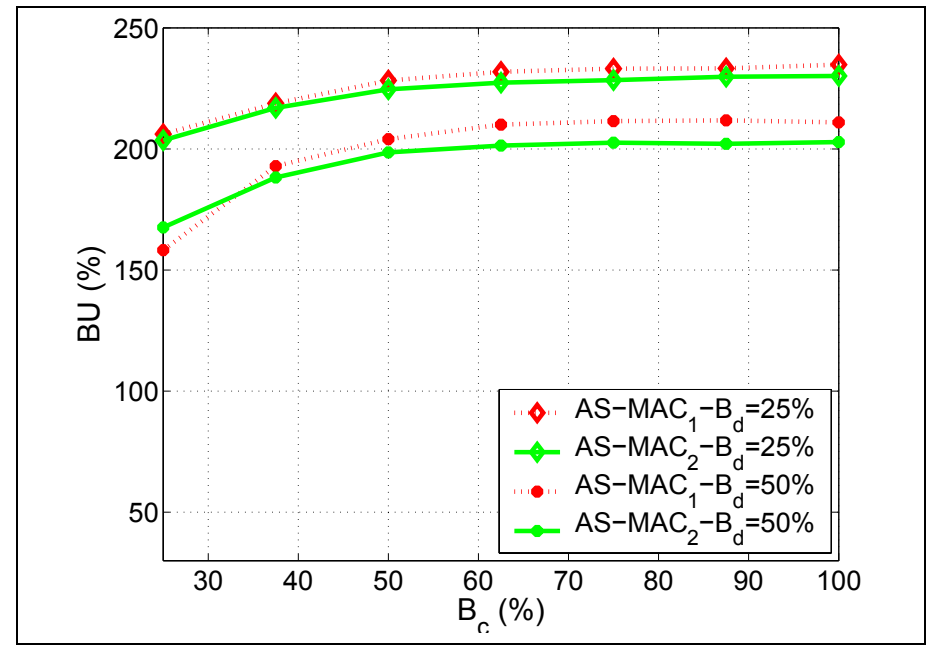

Figure 8. \% $B U$ when two transceivers are used and $B_{d}=25 \%$ and $50 \%$, Vs $B_{c}$

A natural question that now arises is how useful is RES when multi-channel sensing is absent. The result of this scenario is shown in Figure 9. "NS" in the legend refers to no sensing being used. "1Tx" means $A N s$ are equipped with only one transceiver and " $2 \mathrm{Tx}$ " means they have two transceivers with one of them permanently listening to the control channel. It is seen that when sensing is absent, AS_MAC 1 performs better than AS_MAC 2 (52.8\% to $33.6 \%$ for $1 \mathrm{Tx}$ and $131 \%$ to $53 \%$ ). The difference is much more pronounced for the " $2 \mathrm{Tx}$ " as now the control packets are being received effectively. In the absence of sensing, $A N S$ are fully dependent on CTS and RES packets for knowing channel status. When control packets are ignored, nodes end up choosing already busy channels leading to excessive collisions. This confirms that RES is important when sensing is absent but not so otherwise.

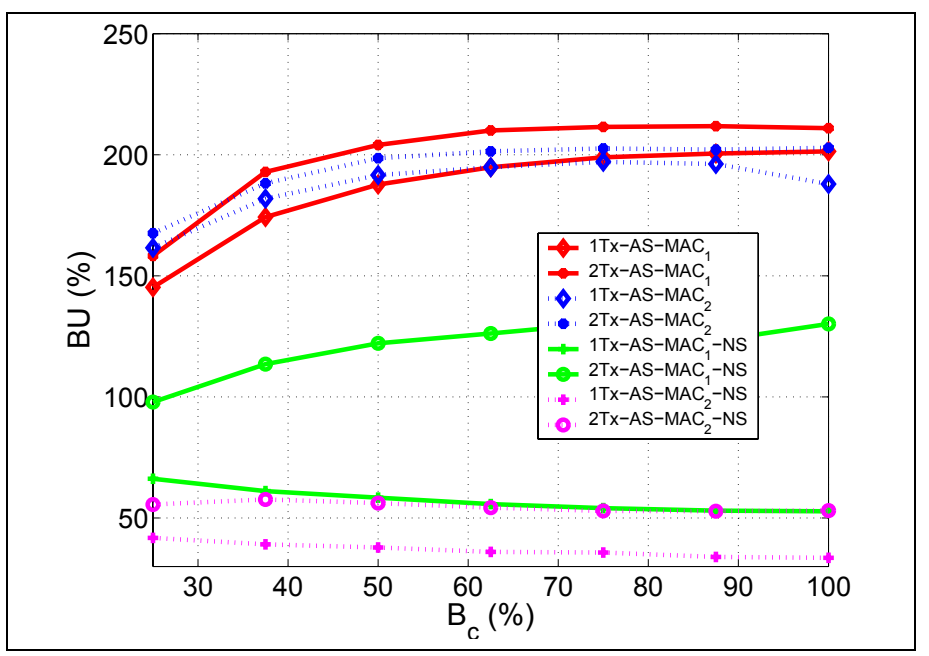

Figure 9. \% $B U$ Vs $B_{c}$ when one or two transceivers are used in the presence and absence of sensing, and $B_{d}$ $\mathbf{5} \mathbf{5 0} \%$

Figure 9 also shows how the presence of sensing helps mitigate MHTP. When ANS use only one transceiver ("1Tx") they suffer from MHTP. It is seen that the performance degradation due to $M H T P$ when sensing is present is much less $\left(211 \%\right.$ to $201 \%$ for $A S_{-} M A C_{1}$, and $203 \%$ to $188 \%$ for $A S_{-} M A C_{2}$ ), while as seen before the performance degradation is much more pronounced when sensing is absent. This illustrates that sensing makes the protocol robust to $M H T P$.

\section{RELATED WORK}

A small number of proposals in the literature have considered PRI-SEC systems. Two models of PRI-SEC interaction are introduced in [3]: the $P R I$ is aware and attempts to accommodate the traffic of the $S E C$, or the $P R I$ has full priority, and it is the responsibility of the $S E C$ to avoid unacceptable levels of interference. The latter model is the one considered here. These two works propose spectrum pooling between a GSM PRI and an OFDM-based WLAN adopting the HIPERLAN standard [12] for the SEC. Our work is significantly different, as we develop an ad hoc $S E C$ system that operates without fixed infrastructure. Moreover, we address a number of practical considerations regarding the interoperation with the PRI GSM, such as the SEC traffic transmission; for example, it is not clear how the $2 m s$ HIPERLAN frames correspond with the GSM slot width of about $0.5 \mathrm{~ms}$. Moreover, our design has the advantage it is not strongly dependent on the physical layer.

Finally, [13] proposes two medium access control protocol designs for a single channel PRI-SEC configuration, assuming that the system has the capability to predict "spectrum holes" which are then used to transfer packets. 
Beyond the different $P R I-S E C$ configuration we consider, our work is not dependent on the prediction of resource availability and thus ensures non-interference between $P R I$ and $S E C$ to the extent that $A N S$ are properly able to sense the spectrum. Moreover, ours is a multi-channel system.

Beyond the PRI-SEC context, a number of MultiChannel MAC (MMAC) protocols were proposed. However, those are either inapplicable or inefficient and thus impractical in the PRI-SEC setting. [7], [8], [9] require that each node is equipped with a number of transceivers equal to number of channels, a clearly impractical assumption. [11] requires three transceivers, while a solution with two transceivers with one of them tuned constantly on the control channel to provide an up-to-date picture of the channels' state was proposed in [5] which uses an additional RES control packet. We have shown that RES is not beneficial in the presence of sensing, thereby reducing control overhead.

The asbsence of upto date channel status information is denoted as the Multi-Channel Hidden Terminal Problem (MHTP) [6] when the protocol operates with a single transceiver and thus alternates between data and control channel transmissions. A solution that alleviates this problem with the requirement that nodes are synchronized is presented in [6]. However, in a multihop setting, as is our $A S N$, the absence of synchronization (non-overlapping 802.11 ATIM windows) renders the scheme unusable. Finally, [10] proposes a single transceiver $M M A C$ protocol, which addresses the MHTP at the expense of network performance. Nodes sense the targeted channels for a period of time equal to the maximum-size frame transmission; if an ACK is received (with $A C K$ 's transmitted on the control channel rather than the data channel), or if the time-out expires the node knows that the channel(s) in question is released and contends for it. The long waiting periods thus introduced would be highly inefficient. This would not be justified in our setting as $A S-M A C$ is already robust to $M H T P$ due to the presence of sensing.

We also briefly note that PRI-SEC systems are fundamentally different from data-over-cellular services, such as $C D P D$ [16] or GPRS. In these cases, the data transmission is actually undertaken by the $P R I$ system while in our case $A S N$ has to provide its service without any help from $P R I$ and as such is much more challenging.

\section{CONCLUSIONS}

We outlined design principles for $A S-M A C$ that enables efficient interworking of GSM and an ad-hoc overlay. Our $A S-M A C$ is shown to improve the overall spectrum utilization by as much as $80 \%$. Our results also indicate that $R E S$ can be safely ignored in the presence of multi-channel sensing, thereby reducing the control overhead. Moreover, the presence of sensing helps overcome MHTP. The insights gained herein are expected to be applicable to general $M M A C$ protocols as well. Thus, we expect channel sensing to play an important role in future systems. Given the large and growing base of deployed cellular infrastructure, it is highly likely that our contributions in the PRI$S E C$ setting we propose will be of immense practical use.

\section{REFERENCES}

[1] http://www.fcc.gov/sptf/files/SEWGFinalReport_1.pdf, $\quad$ FCC Spectrum policy task force, Nov. 2002

[2] Spectrum occupancy measurements by Shared Spectrum Inc., http://www.sharedspectrum.com/Measurements.htm

[3] Capar, F., Martoyo, I., Weiss, T., Jondral, F., K., "Comparison of bandwidth utilization for controlled and uncontrolled channel assignment in a spectrum pooling system”, IEEE VTC, May 2002

[4] Capar, F., Martoyo, I., Weiss. T, and Jondral, F.,K., "Analysis of coexistence strategies for cellular and wireless local area networks", IEEE VTC, Oct. 2003

[5] Wu, S., L., Lin, C., Y., Tseng, Y., C., Sheu, J., L., “A new multichannel MAC protocol with on-demand channel assignment for multi-hop mobile ad hoc networks", International Symposium on Parallel Architectures, Algorithms and Networks, Dec. 2000

[6] So, J., and Vaidya, N., "Multi-channel MAC for ad-hoc networks: Handling multi-channel hidden terminals using a single transceiver", MobiHoc, 2004.

[7] Nasipuri, A., Zhuang, J., and Das, S., R., "A multi-channel CSMA MAC protocol for multi-hop wireless networks", WCNC, Sept. 1999

[8] Nasipuri, A., Das, S., R., "Multi-channel CSMA with signal power-based channel selection for multi-hop wireless networks", IEEE VTC, Sept. 2000

[9] Jain, N., Das, S., R., Nasipuri, A., “A multichannel CSMA MAC protocol with receiver-based channel selection for multi-hop wireless networks", Tenth International Conference on Computer Communications and Networks, Oct. 2001

[10] Choi, N., Seok, Y., Choi, Y., "Multi-channel MAC protocol for mobile ad hoc networks", VTC, Oct. 2003

[11] Pathmasuntharam, J.,S., Das. A, Gupta, A.,K, "Primary channel assignment based MAC (PCAM) - a multi-channel MAC protocol for multi-hop wireless networks", WCNC, Mar. 2004

[12] Weiss, T., A., Jondral, F., K., "Spectrum pooling: an innovative strategy for the enhancement of spectrum efficiency", IEEE Communications Magazine, March 2004,

[13] Syrotiuk, V.,R., Cui, M., Ramkumar, S., Colbourn, C.,J., "Dynamic spectrum utilization in ad-hoc networks", Elsevier Computer Networks Journal, Dec 2004

[14] Mouley, M., and Pautet, M.,B., "The GSM system for mobile communication", Cell \& Systems, Palaiseau, France, 1992

[15] Wireless Network Security Center, Stevens Institute of technology, www.winsec.us

[16] CDPD Forum, "Cellular Digital Packet Data System Specification: Release 1.1", Tech. rep., Jan. 1995

[17] Qualnet Network Simulator by Scalabale network Technologies Inc., www.qualnet.com

[18] ETSI HIPERLAN/2 specifications, http://www.etsi.org/t $\% 5$ Fnews/0005\%5Fbran.htm

[19] Cabric, D., Mishra, S.,M., Brodersen, R.,W., "Implementation Issues in Spectrum Sensing for Cognitive Radios", Asilomar Conference on Signals, Systems, and Computers, 2004

[20] Mukherjee,, T., et al., "Reconfigurable MEMS-enabled RF Circuits for Spectrum Sensing", in Government Microcircuit Applications and Critical Technology Conference, April 2005 